\title{
Wideband DOA Estimation via Sparse Bayesian Learning over a Khatri-Rao Dictionary
}

\author{
Yujian PAN, Ning TAI, Naichang YUAN \\ College of Electronic Science and Engineering, National University of Defense Technology, \\ Changsha, Hunan, 410073, China \\ pyj8711@qq.com
}

\begin{abstract}
This paper deals with the wideband directionof-arrival (DOA) estimation by exploiting the multiple measurement vectors (MMV) based sparse Bayesian learning $(S B L)$ framework. First, the array covariance matrices at different frequency bins are focused to the reference frequency by the conventional focusing technique and then transformed into the vector form. Then a matrix called the Khatri-Rao dictionary is constructed by using the KhatriRao product and the multiple focused array covariance vectors are set as the new observations. DOA estimation is to find the sparsest representations of the new observations over the Khatri-Rao dictionary via SBL. The performance of the proposed method is compared with other wellknown focusing based wideband algorithms and the CramerRao lower bound (CRLB). The results show that it achieves higher resolution and accuracy and can reach the CRLB under relative demanding conditions. Moreover, the method imposes no restriction on the pattern of signal power spectral density and due to the increased number of rows of the dictionary, it can resolve more sources than sensors.
\end{abstract}

\section{Keywords}

Array covariance vector, direction-of-arrival estimation, focusing technique, sparse Bayesian learning, wideband source

\section{Introduction}

Direction of arrival (DOA) estimation is an important part of the array signal processing and widely applied in the civil and military fields, e.g., communication, radar and sonar. Since the narrowband estimation method such as MUSIC and ESPRIT cannot be directly used under the wideband scenarios which commonly exist in practice, researchers have proposed a number of specific methods.

Incoherent subspace method (ISM) [1] is the first proposed method which averages the DOA estimations under different frequency bins in-band. Thus the power variation in-band will deteriorate its performance. Moreover, it cannot deal with the coherent sources. To make up this weakness, the coherent subspace method (CSM) was proposed [2-4]. It averages the array covariance matrices obtained by the focusing technique and then the narrowband DOA estimation method follows. The frequency smoothing involved in that procedure enables it to resolve the coherent sources. Additionally, it improves the angle resolution and the estimation accuracy. However, the CSM needs DOA pre-estimation usually acquired by the traditional beamforming and the inaccuracy in pre-estimation will adversely affect the performance of CSM. Based on CSM, Feng came up with the focusing Khatri-Rao and rotational signal-subspace (FKRRSS) method [5] which transforms the array covariance matrices of different frequency bins into a higher dimensional matrix through Khatri-Rao product. Then DOA estimation is done by the subspace type algorithm. The author claimed that, compared with CSM, the method improved the robustness to the inaccuracy in pre-estimation and the angle resolution. But in that method there is a key assumption that the matrix $\left[\boldsymbol{H}^{T}, \mathbf{1}_{j}\right]$ should be full column rank. ( $\mathbf{1}_{j}$ is the column vector with all entries being one and see below for the meaning of $\boldsymbol{H}$ ). That means, at least, the sources should neither have flat power spectral densities nor have similar power spectral density with each other. This strict assumption will limit its application range. We will show that even if this assumption is satisfied, its performance is not satisfying in our simulation conditions. Another method based on KhatriRao product can be found in [6]. However, the transformed noises at some virtual sensors are coherent and the power compensation to remove power variation in-band can only be applied to signals with similar power spectral densities.

Recently, the sparse representation related to the compressed sensing (CS) and machine learning has found its application in DOA estimation. The narrowband DOA estimation algorithms $\ell 1-S V D$ [7] based on $\ell 1$-norm and RVMDOA [8] based on SBL are the two well-known algorithms. The abbreviations SVD and RVM denotes the singular value decomposition and relevance vector machine, respectively. Their performances both are much better that of the traditional subspace type method. However, $\ell 1-S V D$ is not as good as RVM-DOA since it is biased for closely-spaced signals. Wideband algorithm based on SBL has also be presented in [9]. It directly processes the array outputs in time domain without executing Fourier transform. However, due to the modeling method, it can only exploit the single mea- 
surement vector (SMV) based SBL whose reconstruction performance is worse than the MMV based SBL [10]. Moreover, it requires specific types of signal modulation or preestimated signal power spectral density to estimate the correlation functions for building the dictionary [11] and the estimation error will leads to performance degradation [12].

In this paper, we propose a new wideband DOA estimation method. By using the focusing technique, the array covariance matrices at different frequency bins are focused to the same frequency. Then the vectorization operation instead of the averaging operation in CSM is performed on the focused array covariance matrices to obtain the multiple focused array covariance vectors. By taking advantage of the spatial sparsity property of sources, the focused array covariance vectors can be sparsely represented over the Khatri-Rao dictionary via MMV based SBL to obtain the DOAs. This method can improve the resolution and accuracy compared with other well-known focusing based algorithms under the same preliminary DOAs estimated by the conventional beamforming method and reach the CRLB under the relative demanding scenario. This indicates the new method decreases its sensitivity to the initial DOAs. Further more, the method removes the restriction that the number of sensors has to be larger than the number of sources and imposes no restriction on the pattern of signal power spectral density. The latter means the method can be applied to signals with flat or non-flat, similar or non-similar signal power spectral densities.

Notations used in the paper are introduced as follows. $(\cdot)^{T},(\cdot)^{*}$ and $(\cdot)^{H}$ are denoted as the transpose, conjugate and conjugate transpose operator, respectively. $(\cdot)_{j},(\cdot)_{\cdot j}$ and $(\cdot)_{j, j}$ denote the $j$ th row, $j$ th column and $j$ th diagonal element of a matrix, respectively. $\|\cdot\|_{2}$ denotes the $\ell 2$ norm. $\operatorname{tr}(\cdot),(*)$ and $\otimes$ are the trace, Khatri-Rao product and Kronecker product operator respectively. $\operatorname{vec}(\cdot)$ is the operator that creates a column vector by stacking the column vectors of a matrix below one another. $\mathrm{E}(\cdot)$ denotes the expectation and $\operatorname{diag}(\cdot)$ is to form a diagonal matrix with entries of a vector.

\section{Model Formulation}

Assume $K$ far-field wideband signals from directions $\theta_{k}, k=1, \ldots, K$ impinge on a uniform linear array (ULA) of $M$ elements whose inter-spacing being $d$. The output of $m$ th element at time $t$ can be written as

$$
x_{m}(t)=\sum_{k=1}^{K} s_{k}\left(t-\tau_{m}\left(\theta_{k}\right)\right)+n_{m}(t)
$$

where $\tau_{m}\left(\theta_{k}\right)$ is the propagation delay for the $k$ th signal between the reference point and the $m$ th element. $s_{k}(t)$ is the $k$ th singal. $n_{m}(t)$ is assumed to be the additive white Gaussian noise. As the sources being wideband, the array outputs in time domain are divided into $I$ segments with each for the discrete Fourier transformation (DFT) to obtain the $J$ narrowband measurements. $I$ is called the frequency snapshot number. The DFT outputs of the $i$ th segment can be represented as

$$
\boldsymbol{X}_{i}\left(f_{j}\right)=\boldsymbol{A}\left(f_{j}, \boldsymbol{\theta}\right) \boldsymbol{S}_{i}\left(f_{j}\right)+\boldsymbol{N}_{i}\left(f_{j}\right), \quad j=1, \ldots, J
$$

where $\boldsymbol{S}_{i}\left(f_{j}\right)$ and $\boldsymbol{N}_{i}\left(f_{j}\right)$ are the frequency component of the signal and noise vectors, respectively. $\quad \boldsymbol{A}\left(f_{j}, \boldsymbol{\theta}\right)=\left[\boldsymbol{a}\left(f_{j}, \theta_{1}\right), \ldots, \boldsymbol{a}\left(f_{j}, \theta_{K}\right)\right]$ is the array manifold matrix and $\boldsymbol{a}\left(f_{j}, \theta_{k}\right)=$ $\left[1, e^{-\mathrm{i} 2 \pi f_{j} d \cos \left(\theta_{k}\right) / c}, \ldots, e^{-\mathrm{i} 2 \pi f_{j}(M-1) d \cos \left(\theta_{k}\right) / c}\right]^{T} . \quad c$ denotes the signal propagation speed. For simplicity, $\boldsymbol{A}_{j}(\boldsymbol{\theta})$ and $\boldsymbol{a}_{j}\left(\theta_{k}\right)$ are used to replace $\boldsymbol{A}\left(f_{j}, \boldsymbol{\theta}\right)$ and $\boldsymbol{a}\left(f_{j}, \theta_{k}\right)$ respectively in the rest of the paper. As $\boldsymbol{N}_{i}\left(f_{j}\right)$ can be assumed to be white Gaussian and uncorrelated with signals, the array covariance matrix at $f_{j}$ can be expressed as

$$
\boldsymbol{R}_{j}=\boldsymbol{A}_{j}(\boldsymbol{\theta}) \boldsymbol{P}_{j} \boldsymbol{A}_{j}^{H}(\boldsymbol{\theta})+\sigma_{j}^{2} \boldsymbol{I}
$$

where $\boldsymbol{P}_{j}$ is the signal covariance matrix and $\sigma_{j}{ }^{2}$ is the noise power at frequency $f_{j}$.

In order to remove the dependence of array manifold matrix on frequency, the focusing technique which arose in CSM is adopted. Here the rotation signal-subspace (RSS) [3] focusing matrix $\boldsymbol{T}_{j}$ is utilized to transform (3) into

$$
\boldsymbol{T}_{j} \boldsymbol{R}_{j} \boldsymbol{T}_{j}^{H}=\boldsymbol{A}_{0}(\boldsymbol{\theta}) \boldsymbol{P}_{j} \boldsymbol{A}_{0}^{H}(\boldsymbol{\theta})+\sigma_{j}^{2} \boldsymbol{I}
$$

where

$$
\boldsymbol{T}_{j}=\boldsymbol{V}_{j} \boldsymbol{U}_{j}^{H}
$$

and $\boldsymbol{A}_{0}(\boldsymbol{\theta})$ is the manifold matrix at the center frequency, $\boldsymbol{U}_{j}$ and $\boldsymbol{V}_{j}$ are the left and right singular matrix of $\boldsymbol{A}_{j}\left(\boldsymbol{\theta}_{p}\right) \boldsymbol{A}_{0}^{H}\left(\boldsymbol{\theta}_{p}\right)$, respectively. $\boldsymbol{\theta}_{p}$ is the preliminary DOAs.

In practice, $\boldsymbol{R}\left(f_{j}\right)$ is consistently estimated by

$$
\hat{\boldsymbol{R}}_{j}=\sum_{i=1}^{I} \boldsymbol{X}_{i}\left(f_{j}\right) \boldsymbol{X}_{i}{ }^{H}\left(f_{j}\right) / I .
$$

As $I$ cannot be infinitely large, $\Delta \boldsymbol{R}_{j}=\hat{\boldsymbol{R}}_{j}-\boldsymbol{R}_{j}$ which is called the covariance estimation error will exist. Denote

$$
\hat{\sigma}_{j}^{2}=\frac{1}{M-K} \sum_{m=K+1}^{M} \rho_{m}
$$

as the estimation of $\sigma_{j}{ }^{2}$ and $\rho_{m}$ is the $m$ th largest eigenvalue of $\hat{\boldsymbol{R}}_{j}$. Then (4) can be turned into

$$
\begin{aligned}
\boldsymbol{T}_{j} \hat{\boldsymbol{R}}_{j} \boldsymbol{T}_{j}^{H}-\hat{\sigma}_{j}^{2} \boldsymbol{I}= & \boldsymbol{A}_{0}(\boldsymbol{\theta}) \boldsymbol{P}_{j} \boldsymbol{A}_{0}^{H}(\boldsymbol{\theta}) \\
& +\boldsymbol{T}_{j} \Delta \boldsymbol{R}_{j} \boldsymbol{T}_{j}^{H}
\end{aligned}
$$

Assume the signals are uncorrelated with each other, and then $\boldsymbol{P}_{j}$ is a diagonal matrix and we can form the focused covariance vector as

$$
\begin{aligned}
\hat{\boldsymbol{y}}_{j} & =\operatorname{vec}\left(\boldsymbol{T}_{j} \hat{\boldsymbol{R}}_{j} \boldsymbol{T}_{j}^{H}-\hat{\sigma}_{j}^{2} \boldsymbol{I}\right) \\
& =\operatorname{vec}\left(\boldsymbol{A}_{0}(\boldsymbol{\theta}) \boldsymbol{P}_{j} \boldsymbol{A}_{0}^{H}(\boldsymbol{\theta})+\boldsymbol{T}_{j} \Delta \boldsymbol{R}_{j} \boldsymbol{T}_{j}^{H}\right) \\
& =\boldsymbol{B} \boldsymbol{\eta}_{j}+\boldsymbol{\varepsilon}_{j}, \quad j=1, \ldots, J
\end{aligned}
$$

where $\boldsymbol{B}=\boldsymbol{A}_{0}^{*}(\boldsymbol{\theta}) * \boldsymbol{A}_{0}(\boldsymbol{\theta})=\left[\boldsymbol{b}\left(\theta_{1}\right), \ldots, \boldsymbol{b}\left(\theta_{K}\right)\right] \in$ $\mathcal{C}^{M^{2} \times K}, \boldsymbol{\eta}_{j}=\left[\eta_{1 j}, \ldots, \eta_{K j}\right]^{T}$ denotes the vector composed of the signals power at frequency $f_{j}$ and $\varepsilon_{j}$ is viewed as the 
error component. According to the rule of Khatri-Rao product, $\boldsymbol{b}\left(\theta_{k}\right)=\boldsymbol{a}_{0}^{*}\left(\theta_{k}\right) \otimes \boldsymbol{a}_{0}\left(\theta_{k}\right)$.

Since $\operatorname{vec}\left(\Delta \boldsymbol{R}_{j}\right)$ approximately follows the complex normal distribution which is $\operatorname{vec}\left(\Delta \boldsymbol{R}_{j}\right) \sim \mathcal{C N}\left(0, \boldsymbol{R}^{T}{ }_{j} \otimes\right.$ $\left.\boldsymbol{R}_{j} / I\right)[13,14]$ and $\varepsilon_{j}=\operatorname{vec}\left(\boldsymbol{T}_{j} \Delta \boldsymbol{R}_{j} \boldsymbol{T}_{j}^{H}\right)=\left(\boldsymbol{T}_{j}^{*} \otimes\right.$ $\left.\boldsymbol{T}_{j}\right) \operatorname{vec}\left(\Delta \boldsymbol{R}_{j}\right)$ [15], we have

$$
\varepsilon_{j} \sim \mathcal{C N}\left(0, Q_{j}\right), \quad j=1, \ldots, J
$$

where

$$
\begin{aligned}
\boldsymbol{Q}_{j} & =\left(\boldsymbol{T}_{j}^{*} \otimes \boldsymbol{T}_{j}\right) \mathrm{E}\left(\operatorname{vec}\left(\Delta \boldsymbol{R}_{j}\right) \operatorname{vec}\left(\Delta \boldsymbol{R}_{j}\right)^{H}\right)\left(\boldsymbol{T}_{j}^{*} \otimes \boldsymbol{T}_{j}\right)^{H} \\
& =\left(\boldsymbol{T}_{j} \boldsymbol{R}_{j} \boldsymbol{T}_{j}^{H}\right)^{T} \otimes\left(\boldsymbol{T}_{j} \boldsymbol{R}_{j} \boldsymbol{T}_{j}^{H}\right) / I
\end{aligned}
$$

Setting $\hat{\boldsymbol{Y}}=\left[\hat{\boldsymbol{y}}_{1}, \ldots, \hat{\boldsymbol{y}}_{J}\right] \in \mathcal{C}^{M^{2} \times J}$, (9) can be extended to a MMV model as

$$
\hat{\boldsymbol{Y}}=\boldsymbol{B} \boldsymbol{H}+\boldsymbol{E}
$$

where $\boldsymbol{H}=\left[\boldsymbol{\eta}_{1}, \ldots, \boldsymbol{\eta}_{J}\right] \in \mathcal{C}^{K \times J}$ and $\boldsymbol{E}=\left[\boldsymbol{\varepsilon}_{1}, \ldots, \boldsymbol{\varepsilon}_{J}\right]$ is the error component that follows the Guassian distribution.

\section{Sparse Bayesian Learning for Wideband DOA Estimation}

In order to adopt the sparse reconstruction algorithm, let $\Theta=\left\{\tilde{\theta}_{l}\right\}_{l=1}^{L}(L \gg M)$ be the uniform sampling grids in the azimuth range $\left[0^{\circ}, 180^{\circ}\right]$ with the grid interval $\Delta \tilde{\theta}$. Then (12) can be reformulated as

$$
\hat{\boldsymbol{Y}}=\boldsymbol{B}(\boldsymbol{\Theta}) \tilde{\boldsymbol{H}}+\boldsymbol{E}
$$

where $\boldsymbol{B}(\boldsymbol{\Theta})=\left[\boldsymbol{b}\left(\theta_{1}\right), \ldots, \boldsymbol{b}\left(\theta_{L}\right)\right] \in \mathcal{C}^{M^{2} \times L}$ is called the Khatri-Rao dictionary since it obtained via the Khatri-Rao product and $\tilde{\boldsymbol{H}}$ is the matrix with all rows being zero except those corresponding to the true DOAs, which indicates each column of $\tilde{\boldsymbol{H}}$ has the same sparsity profile.

As elements of $\tilde{\boldsymbol{H}}$ denote signal power, it should be a non-negative matrix. Assigning it a truncated normal distribution is reasonable [16], however, it will largely increase the computation cost [17]. So we follow the standard SBL procedure $[10,18]$ and assign it with a normal distributed prior as $\mathrm{p}(\tilde{\boldsymbol{H}} \mid \gamma)=\prod_{j=1}^{j} \mathcal{C N}\left(\tilde{\boldsymbol{H}}_{\cdot j} \mid 0, \boldsymbol{\Gamma}\right)$ where $\boldsymbol{\Gamma}=\operatorname{diag}(\boldsymbol{\gamma}), \boldsymbol{\gamma}=\left[\gamma_{1}, \ldots, \gamma_{L}\right]^{T}$ is the unknown hyperparameter. From (11) we find the error components generally have different covariances at different frequency bins. However, if we model $\mathrm{p}(\hat{\boldsymbol{Y}} \mid \tilde{\boldsymbol{H}})=$ $\prod_{j=1}^{J} \mathcal{C N}\left(\hat{\boldsymbol{Y}}_{\cdot j} \mid \boldsymbol{B}(\boldsymbol{\Theta}) \tilde{\boldsymbol{H}}_{\cdot j}, \boldsymbol{Q}_{j}\right)$, the eventual DOA estimation will be the average of estimations under different single measurement vector (SMV) model, which will deteriorate the performance. So we alter to $\mathrm{p}(\hat{\boldsymbol{Y}} \mid \tilde{\boldsymbol{H}})=$ $\prod_{j=1}^{J} \mathcal{C N}\left(\hat{\boldsymbol{Y}}_{\cdot j} \mid \boldsymbol{B}(\boldsymbol{\Theta}) \tilde{\boldsymbol{H}}_{\cdot j}, \hat{\boldsymbol{Q}}\right)$ where $\hat{\boldsymbol{Q}}=\sum_{j=1}^{J} \hat{\boldsymbol{Q}}_{j} / J$ and $\hat{\boldsymbol{Q}}_{j}=\left(\boldsymbol{T}_{j} \hat{\boldsymbol{R}}\left(f_{j}\right) \boldsymbol{T}_{j}^{H}\right)^{T} \otimes\left(\boldsymbol{T}_{j} \hat{\boldsymbol{R}}\left(f_{j}\right) \boldsymbol{T}_{j}^{H}\right) / I$. This alteration makes sense with sources having flat power spectrum ${ }^{1}$, which does not mean our method can only be applied in this situation since [19] concluded that the optimal performance of SBL cannot be obtained with the true covariance of error component. Thus, in Section 4, one can notice that our method can also gain high performance with sources having non-flat power spectrum.

According to the Bayesian rule the posterior distribution of $\tilde{\boldsymbol{H}}$ can be written as $\mathrm{p}(\tilde{\boldsymbol{H}} \mid \hat{\boldsymbol{Y}})=$ $\prod_{j=1}^{J} \mathcal{C N}\left(\tilde{\boldsymbol{H}}_{\cdot j} \mid \mathcal{M}_{\cdot j}, \boldsymbol{\Sigma}\right)$ with the mean

$$
\mathcal{M}=\boldsymbol{\Gamma} \boldsymbol{B}^{H}(\boldsymbol{\Theta}) \boldsymbol{\Sigma}_{\hat{Y}}^{-1} \hat{\boldsymbol{Y}}
$$

and the covariance

$$
\boldsymbol{\Sigma}=\boldsymbol{\Gamma}-\boldsymbol{\Gamma} \boldsymbol{B}^{H}(\boldsymbol{\Theta}) \boldsymbol{\Sigma}_{\hat{Y}}^{-1} \boldsymbol{B}(\boldsymbol{\Theta}) \boldsymbol{\Gamma}
$$

where $\boldsymbol{\Sigma}_{\hat{Y}}=\hat{\boldsymbol{Q}}+\boldsymbol{B}(\boldsymbol{\Theta}) \boldsymbol{\Gamma} \boldsymbol{B}^{H}(\boldsymbol{\Theta})$. To find the unknown hyperparameter, we need to adopt the type-II maximum likelihood method which indicates to maximize $\mathrm{p}(\hat{\boldsymbol{Y}} \mid \gamma)=$ $\int \mathrm{p}(\hat{\boldsymbol{Y}} \mid \tilde{\boldsymbol{H}}) \mathrm{p}(\tilde{\boldsymbol{H}} \mid \gamma) d \tilde{\boldsymbol{H}}$. Finally, it is equivalent to minimize

$$
L(\gamma)=\ln \left|\boldsymbol{\Sigma}_{\hat{Y}}\right|+\operatorname{tr}\left(\boldsymbol{\Sigma}_{\hat{Y}}^{-1} \hat{\boldsymbol{R}}_{\hat{\boldsymbol{Y}}}\right)
$$

where $\hat{\boldsymbol{R}}_{\hat{\boldsymbol{Y}}}=\hat{\boldsymbol{Y}} \hat{\boldsymbol{Y}}^{H} / J$. The Expectation-Maximization (EM) algorithm can be used to solve the problem by treating $\tilde{\boldsymbol{H}}$ as the hidden variable. However, to speed up convergence, the fixed-point iteration is introduced as following $[10,18]$,

$$
\gamma_{l}^{(q+1)}=\frac{\left\|\mathcal{M}_{j .}\right\|_{2}^{2}}{J\left(1-\boldsymbol{\Sigma}_{l, l} / \gamma_{l}^{(q)}\right)}+\varsigma, \quad l=1, \ldots, L
$$

where $\gamma_{l}(q)$ is the value at $q$ th iteration and $\varsigma$ is a small positive constant to prevent division by zero. The iteration process is terminated if $\left\|\gamma^{(q+1)}-\gamma^{(q)}\right\|_{2} /\left\|\gamma^{(q)}\right\|_{2}<\tau$ where $\tau$ is the termination threshold.

Denote $\hat{\gamma}$ as the estimation of $\gamma$ after iteration converged. If the true DOAs are fortunately at the spatial sampling grids, the peak locations of $\hat{\gamma}$ can be directly output as DOA estimations, or the grid mismatch will happen. One solution is to increase the number of grids adaptively during the iteration [7]. But here we perform a post-processing whose computational cost is lower [8]. Denote $\boldsymbol{\theta}_{k}$ as the set which consists of two adjacent grids relating to the $k$ th peak location of $\hat{\gamma}, \Theta_{-k}$ as the grid set obtained by removing $\boldsymbol{\theta}_{k}$ from $\Theta, \hat{\boldsymbol{\Gamma}}_{-k} \in \mathcal{C}^{(L-2) \times(L-2)}$ as the matrix which removes the diagonal elements corresponding to $\boldsymbol{\theta}_{k}$ from $\hat{\boldsymbol{\Gamma}}$ where $\hat{\boldsymbol{\Gamma}}=\operatorname{diag}(\hat{\gamma}), \boldsymbol{\Sigma}_{-k}=\hat{\boldsymbol{Q}}+\boldsymbol{B}\left(\boldsymbol{\Theta}_{-k}\right) \hat{\boldsymbol{\Gamma}}_{-k} \boldsymbol{B}^{H}\left(\boldsymbol{\Theta}_{-k}\right)$. According to (16), the method is to adjust $\beta_{k}$ and $\theta$ to minimize

$$
\begin{aligned}
L\left(\beta_{k}, \theta\right)= & \ln \left|\boldsymbol{\Sigma}_{-k}+\beta_{k} \boldsymbol{b}(\theta) \boldsymbol{b}^{H}(\theta)\right| \\
& +\operatorname{tr}\left(\left[\boldsymbol{\Sigma}_{-k}+\beta_{k} \boldsymbol{b}(\theta) \boldsymbol{b}^{H}(\theta)\right]^{-1} \hat{\boldsymbol{R}}_{\hat{\boldsymbol{Y}}}\right)
\end{aligned}
$$

where $\beta_{k}$ is a fitting parameter. Taking the derivative with respect to $\beta_{k}$ and $\theta$, equating to zero, we finally achieve the post-processing rule as

$$
\begin{gathered}
\theta_{r k}=\arg \max _{\theta \in \Omega_{k}} \mid \operatorname{Re}\left[\boldsymbol { b } ^ { H } ( \theta ) \boldsymbol { \Sigma } _ { - k } ^ { - 1 } \left(\boldsymbol{b}(\theta) \boldsymbol{b}^{H}(\theta) \boldsymbol{\Sigma}_{-k}^{-1} \hat{\boldsymbol{R}}_{\hat{\boldsymbol{Y}}}\right.\right. \\
\left.\left.-\hat{\boldsymbol{R}}_{\hat{\boldsymbol{Y}}} \boldsymbol{\Sigma}_{-k}^{-1} \boldsymbol{b}(\theta) \boldsymbol{b}^{H}(\theta)\right) \boldsymbol{\Sigma}_{-k}^{-1} \frac{\partial \boldsymbol{b}(\theta)}{\partial \theta}\right]\left.\right|^{-1} \\
k=1, \ldots, K
\end{gathered}
$$

\footnotetext{
${ }^{1}$ The alteration can make sense with the two-sided correlation transformation (TCT) focusing matrix [4] without the limitation of signal pattern. But we empirically find applying TCT focusing matrix in our method cannot get good performance.
} 
where $\Omega_{k}$ is the interval between the grids corresponding to $\boldsymbol{\theta}_{k}$. The post-processing is to scan within $\Omega_{k}$ with a small step size for every source.

In the following, the steps of the proposed wideband DOA estimation algorithm can be summarized as

1. Divide the array outputs into $I$ segments with each for DFT and then obtain $\hat{\boldsymbol{R}}\left(f_{j}\right), j=1, \ldots, J$ by (6).

2. Perform conventional beamforming (CBF) on $\hat{\boldsymbol{R}}\left(f_{0}\right)$ to pre-estimate the DOA and output the pre-estimation as $\boldsymbol{\theta}_{p}=\left[\boldsymbol{\theta}_{C B F}-\theta_{B W} / 4, \boldsymbol{\theta}_{C B F}, \boldsymbol{\theta}_{C B F}+\theta_{B W} / 4\right]\left(\theta_{B W}\right.$ is the beamwidth of the array) [3] and acquire the focusing matrix $\boldsymbol{T}_{j}, j=1, \ldots, J$ by (5).

3. Form the multiple focused array covariance vectors $\hat{\boldsymbol{Y}}=\left[\hat{\boldsymbol{y}}_{1}, \ldots, \hat{\boldsymbol{y}}_{J}\right]$ by (7) and (9).

4. Follow the SBL method to iterate between (14) (15) and (17) with the initialization of $\gamma^{(0)}=$ $\sum_{j=1}^{J}\left|\boldsymbol{B}^{H}(\boldsymbol{\Theta}) \hat{\boldsymbol{Y}}_{\cdot j}\right| / J$ to yield $\hat{\boldsymbol{\gamma}}$.

5. For each peak of $\hat{\gamma}$, execute the post-processing by (19) to output the ultimate DOA estimations.

It should be noticed that the Khatri-Rao Dictionary $\boldsymbol{B}$ has $M^{2}$ rows, which indicates the proposed method has the potential to detect more sources than sensors. Theoretically, with a minimum redundancy linear array (MRLA) it can resolve $\left(M^{2}-M\right) / 2$ sources simultaneously at most. Subtracting $M$ is to delete the diagonal elements from the covariance matrix since they are the same in noiseless situation and division by two is to remove the symmetric information since the covariance matrix is Hermitian.

Regarding the computational complexity of the proposed method, the computational cost mainly lies in the SBL iteration process in step (4). Assuming $L>M \sqrt{J}$ and $L>M^{2}$, the computational complexity of SBL is $O\left(L^{2} M^{2}\right)$ per iteration. However, since we adopt the fixedpoint iteration and do not need to update the noise variance, the iteration converges much faster than the stand SBL method in [10].

\section{Simulation Results}

In this section, some experiments are performed to evaluate the performance of our algorithm. An ULA of five elements is exposed to two uncorrelated wideband sources. The intersensor spacing is half wavelength at the center frequency of $100 \mathrm{MHz}$ and the relative bandwidth of signals are $20 \%$. The array outputs are decomposed into 9 narrowband components in each segment (with 16 point fast Fourier transform (FFT)), i.e., $J=9$. The positive constant in (17) and the iteration termination threshold are set as $\varsigma=10^{-10}$ and $\tau=10^{-4}$, respectively. The maximum iteration times is limited as 2000. The grid interval is $1^{\circ}$ (i.e., $L=181$ ) and the scanning step size is $0.1^{\circ}$. In order to take into account the impact of pre-estimation on the performance, the pre-estimated DOAs are obtained by CBF in every single trial [20]. The simulation results are obtained by averaging 300 Monte Carlo trials. The RSS, TCT and FKR-RSS method are chosen as the comparative algorithms. The wideband CRLB whose detailed derivation can be seen in [21] is also included. In order to make FKR-RSS work normally, the sources with non-flat power spectrum are selected to insure $\left[\boldsymbol{H}^{T}, \mathbf{1}_{J}\right]$ being full column rank.

Firstly, we simulate the performance versus signalto-noise ratio (SNR). The DOAs of sources are set as $\left[85.2^{\circ}, 94.7^{\circ}\right]$ with the frequency snapshot number being 50 . The results are depicted in Fig. 1. The vertical axis represents the root-mean-square error (RMSE) between the estimated value and true value. Compared to the other methods, our method achieves the highest DOA estimation accuracy and reaches the $\mathrm{CRLB}$ at $\mathrm{SNR}=-4 \mathrm{~dB}$. Meanwhile, the FKR-RSS only surpasses the traditional methods under low SNR. The results here have confirmed our previous perspective that the new proposed method can also work with sources having non-flat power spectrum. Additionally, although there are errors in the preliminary DOAs, our method arrives the CRLB. It indicates that in the new method the sensitivity to the initial DOAs is decreased.

Secondly, we address the estimation performance under different frequency snapshot number. The simulation conditions are the same as above except for SNR $=0 \mathrm{~dB}$. The results are revealed in Fig. 2, where we can notice that when the frequency snapshot number is as low as 20, our method reaches the CRLB. The FKR-RSS gets the similar performance as in Fig. 1. It exceeds the traditional methods with small frequency snapshot number and falls behind when snapshot number becomes larger.

Thirdly, we vary the angle separation to evaluate angle resolution performance. $\mathrm{SNR}=0 \mathrm{~dB}$ and the DOAs of sources are set as $\left[85.2^{\circ}, 85.2^{\circ}+\Delta \theta\right]$ where $\Delta \theta$ is the angle separation. Figure 3 demonstrates the results. Compared to the other methods, the proposed method possesses the highest angle resolution. The proposed method achieves the es-

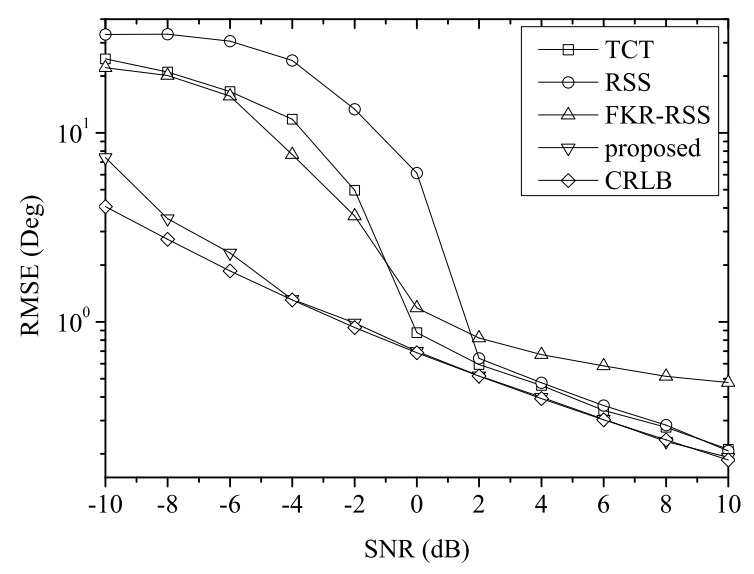

Fig. 1. RMSE of wideband DOA estimation versus SNR. 


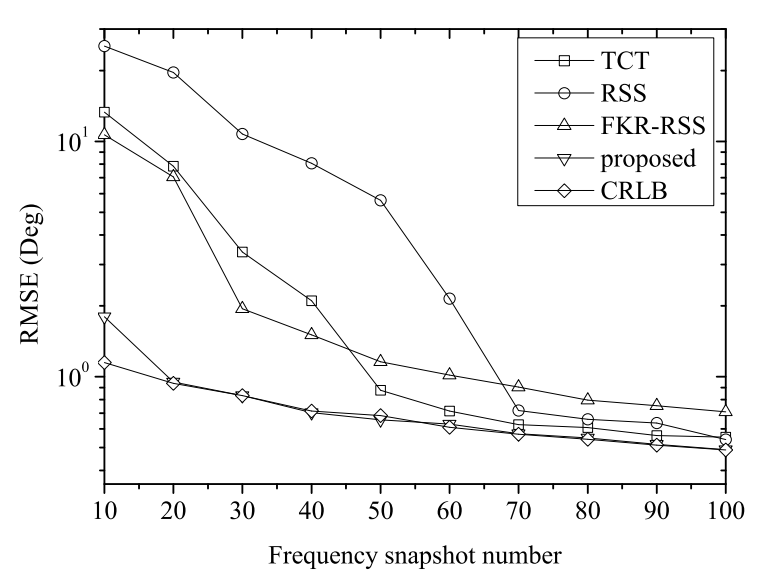

Fig. 2. RMSE of wideband DOA estimation versus frequency snapshot number.

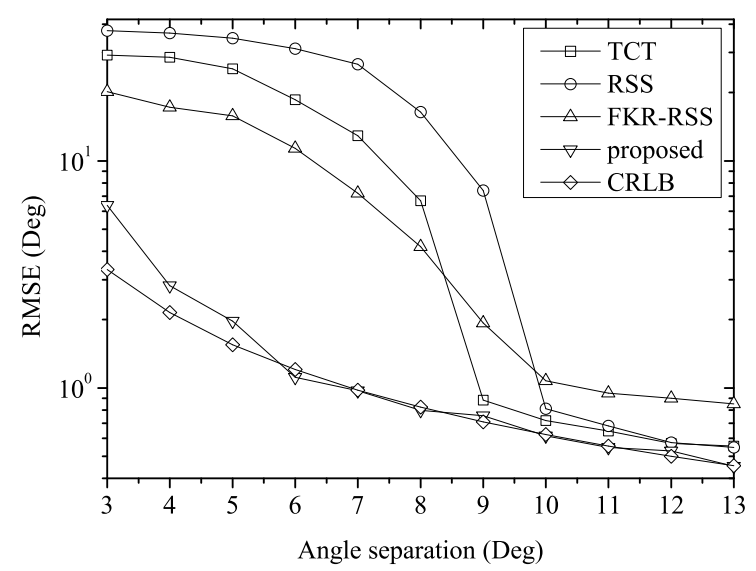

Fig. 3. RMSE of wideband DOA estimation versus angle separation.

timation error of no more than $3^{\circ}$ under angle separation of larger than $4^{\circ}$ while other methods reach this performance under angle separation of at least larger than $8^{\circ}$. In addition, when angle separation is larger than $6^{\circ}$, our method arrives the CRLB.

At last, we demonstrate the ability to resolve more sources than sensors. A four elements MRLA whose elements locate at $[0, \lambda, 2.5 \lambda, 3 \lambda]$ is exposed to six sources from the direction of $\left[51^{\circ}, 60^{\circ}, 83^{\circ}, 93^{\circ}, 115^{\circ}, 125^{\circ}\right] . \lambda$ is the wavelength corresponding to the center frequency. The simulation condition is the same as the previous except for $\mathrm{SNR}=10 \mathrm{~dB}$. The noise variance is assumed to be known and the preliminary DOAs are given by step (2) in Section 3. The spatial spectrum is shown in Fig. 4 . It can be seen that all sources are correctly resolved.

\section{Conclusion}

Wideband DOA estimation is more complex than the narrowband counterpart. In this paper, we estimate the DOAs by exploiting the MMV based SBL framework and sparsely representing the focused array covariance vectors over the Khatri-Rao dictionary. The simulation results

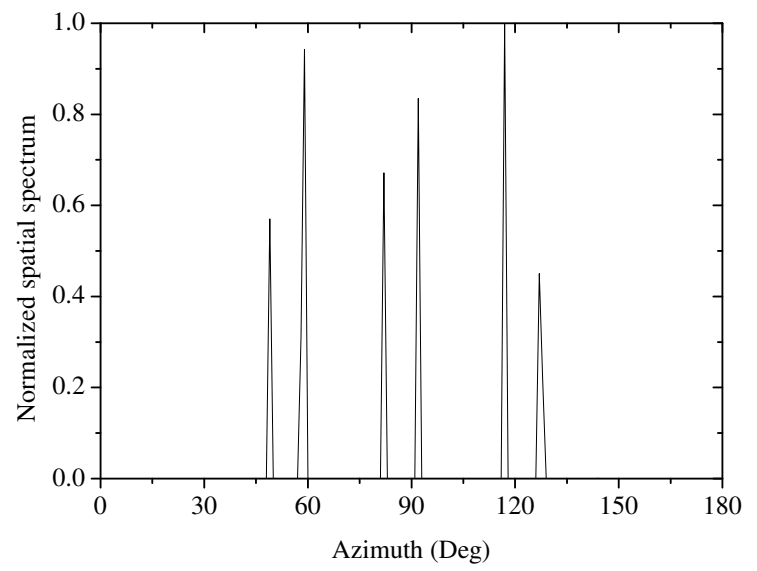

Fig. 4. Resolving six sources with four sensors.

demonstrate several advantages of the new method, e.g., higher resolution and accuracy, reaching the CRLB under relative demanding scenario, imposing no restriction on the pattern of signal power spectral density and resolving more sources than sensors. However, due to the way of model formulation the correlated scenario, especially coherent, will lead to a deteriorated performance. Possible future work might concern the extension the proposed method to the above situation.

\section{Acknowledgments}

This work was supported in part by the National Natural Science Foundation of China under Grant no. 61302141. The authors would like to thank the anonymous reviewers for the improvement of this paper.

\section{References}

[1] WAX, M., SHAN, T.-J., KAILATH, T. Spatio-temporal spectral analysis by eigenstructure methods. IEEE Transactions on Acoustics, Speech, and Signal Processing, 1984, vol. 32, no. 4, p. 817-827. DOI: 10.1109/TASSP.1984.1164400

[2] WANG, H., KAVEH, M. Coherent signal-subspace processing for the detection and estimation of angles of arrival of multiple wide-band sources. IEEE Transactions on Acoustics, Speech, and Signal Processing, 1985, vol. 33, no. 4, p. 823-831. DOI: 10.1109/TASSP.1985.1164667

[3] HUNG H., KAVEH, M. Focussing matrices for coherent signalsubspace processing. IEEE Transactions on Acoustics, Speech, and Signal Processing, 1988, vol. 36, no. 8, p. 1272-1281. DOI: $10.1109 / 29.1655$

[4] VALAEE, S., KABAL, P. Wideband array processing using a twosided correlation transformation. IEEE Transactions on Signal Processing, 1995, vol. 43, no. 1, p. 160-172. DOI: 10.1109/78.365295

[5] FENG, D., BAO, M., YE, Z., GUAN, L., LI, X. A novel wideband DOA estimator based on khatri-rao subspace approach. Signal Processing, 2011, vol. 91, no. 10, p. 2415-2419. DOI: 10.1016/j.sigpro.2011.04.014 
[6] YAMADA H., SHIRAI S., YAMAGUCHI, Y. DOA Estimation of wideband signals using extended virtual array. In Proceedings of $3^{\text {rd }}$ Asia-Pacific Conference on Antennas and Propagation. Harbin (China), 2014, p. 802-805. DOI: 10.1109/APCAP.2014.6992620

[7] MALIOUTOV, D., CETIN, M., WILLSKY, A. S. A sparse signal reconstruction perspective for source localization with sensor arrays. IEEE Transactions on Signal Processing, 2005, vol. 53, no. 8, p. 3010-3022. DOI: 10.1109/TSP.2005.850882

[8] LIU, Z. M., HUANG, Z. T., ZHOU, Y. Y. An efficient maximum likelihood method for direction-of-arrival estimation via sparse Bayesian learning. IEEE Transactions on Wireless Communications, 2012, vol. 11, no. 10, p. 3067-3617. DOI: 10.1109/TWC.2012.090312.111912

[9] LIU, Z. M., HUANG, Z. T., ZHOU, Y. Y. Sparsity-inducing direction finding for narrowband and wideband signals based on array covariance vector. IEEE Transactions on Wireless Communications, 2013, vol. 12, no. 8, p. 3896-3907. DOI: 10.1109/TWC.2013.071113.121305

[10] WIPF, D. P., RAO, B. D. An empirical Bayesian strategy for solving the simultaneous sparse approximation problem. IEEE Transactions on Signal Processing, 2007, vol. 55, no. 7, p. 3704-3716. DOI: 10.1109/TSP.2007.894265

[11] LIU, Z. M., HUANG, Z. T., ZHOU, Y. Y. Direction-of-Arrival estimation of wideband signals via covariance matrix sparse representation. IEEE Transactions on Signal Processing, 2011, vol. 59, no. 9, p. 4256-4270. DOI: 10.1109/TSP.2011.2159214

[12] GAN, L., WANG, X. DOA estimation of wideband signals based on slice-sparse representation. EURASIP Journal on Advances in Signal Processing, 2013, vol. 2013, no. 18, p. 1-10. DOI: 10.1186/16876180-2013-18

[13] YIN, J., CHEN, T. Direction-of-arrival estimation using a sparse representation of array covariance vectors. IEEE Transactions on Signal Processing, 2011, vol. 59, no. 9, p. 4489-4493. DOI: 10.1109/TSP.2011.2158425

[14] OTTERSTEN, B., STOICA, P., ROY, R. Covariance matching estimation techniques for array signal processing applications. Digital Signal Processing, 1998, vol. 8, no. 3, p. 185-210. DOI: 10.1006/dspr.1998.0316

[15] MAGNUS, J. R., NEUDECKER, H. Matrix Differential Calculus with Applications in Statistics and Econometrics. $3^{\text {nd }}$ ed. Chichester: Wiley, 1988.

[16] ROBERT, C. P. Simulation of truncated normal variables. Statistics and Computing, 1995, vol. 5, no. 2, p. 121-125. DOI: 10.1007/BF00143942

[17] THEMELIS, K. E., RONTOGIANNIS, A. A., KOUTROUMBAS, K. D. A novel hierarchical Bayesian approach for sparse semisupervised hyperspectral unmixing. IEEE Transactions on
Signal Processing, 2012, vol. 60, no. 2, p. 585-599. DOI: 10.1109/TSP.2011.2174052

[18] TIPPING, M. E. Sparse Bayesian learning and the relevance vector machine. Journal of Machine Learning Research, 2001, vol. 1, p. $211-244$.

[19] ZHANG, Z., RAO, B. Clarify Some Issues on the Sparse Bayesian Learning for Sparse Signal Recovery, technical report. San Diego (CA, USA): University of California. [Online] Cited 2011. Available at: https://sites.google.com/site/researchbyzhang/.

[20] YOON, Y. S., KAPLAN, L. M., MCCLELLAN, J. H. Tops: new DOA estimator for wideband signals. IEEE Transactions on Signal Processing, 2006, vol. 54, no. 6, p. 1977 - 1989. DOI: 10.1109/TSP.2006.872581

[21] FRIEDLANDER, B., WEISS, A. Direction finding for wide-band signals using an interpolated array. IEEE Transactions on Signal Processing, 1993, vol. 41, no. 4, p. 1618-1634. DOI: 10.1109/78.212735

\section{About the Authors ...}

Yujian PAN was born in 1987. He received his M.S. degree in Electronic Science and Technology from National University of Defense Technology in 2012. Currently he is working towards the Ph.D. degree in College of Electronic Science and Engineering, National University of Defense Technology, Changsha, Hunan, China. His research interests include array signal processing and microwave circuit design.

Ning TAI was born in 1989. He received his M.S. degree in Electronic Science and Technology from National University of Defense Technology in 2013. Currently he is working towards the $\mathrm{PhD}$ degree in College of Electronic Science and Engineering, National University of Defense Technology, Changsha, Hunan, China. His research interests include radar signal processing and radar system simulation.

Naichang YUAN was born in 1965. He received his M.S. and Ph.D. degree in Electronic Science and Technology from University of Electronic Science and Technology of China in 1991 and 1994, respectively. He is currently a professor with the College of Electronic Science and Engineering, National University of Defense Technology, Changsha, Hunan, China. His research interests include array signal processing, SAR imaging processing and singal processing in radar. 